\title{
Postselection-Loophole-Free Bell Violation with Genuine Time-Bin Entanglement
}

\author{
Francesco Vedovato, ${ }^{1,2}$ Costantino Agnesi, ${ }^{1}$ Marco Tomasin, ${ }^{1}$ Marco Avesani,,${ }^{1}$ Jan-Åke Larsson, ${ }^{3}$ \\ Giuseppe Vallone, ${ }^{1,4}$ and Paolo Villoresi ${ }^{1,4}$ \\ ${ }^{1}$ Dipartimento di Ingegneria dell'Informazione, Università di Padova, via Gradenigo 6B, 35131 Padova, Italy \\ ${ }^{2}$ Centro di Ateneo di Studi e Attività Spaziali "G. Colombo", Università di Padova, via Venezia 15, 35131 Padova, Italy \\ ${ }^{3}$ Institutionen för systemteknik, Linköping Universitet, 58183 Linköping, Sweden \\ ${ }^{4}$ Istituto di Fotonica e Nanotecnologie, CNR, via Trasea 7, 35131 Padova, Italy
}

(Received 29 May 2018; revised manuscript received 11 September 2018; published 9 November 2018)

\begin{abstract}
Entanglement is an invaluable resource for fundamental tests of physics and the implementation of quantum information protocols such as device-independent secure communications. In particular, time-bin entanglement is widely exploited to reach these purposes both in free space and optical fiber propagation, due to the robustness and simplicity of its implementation. However, all existing realizations of time-bin entanglement suffer from an intrinsic postselection loophole, which undermines their usefulness. Here, we report the first experimental violation of Bell's inequality with "genuine" time-bin entanglement, free of the postselection loophole. We introduced a novel function of the interferometers at the two measurement stations, that operate as fast synchronized optical switches. This scheme allowed us to obtain a postselection-loophole-free Bell violation of more than 9 standard deviations. Since our scheme is fully implementable using standard fiberbased components and is compatible with modern integrated photonics, our results pave the way for the distribution of genuine time-bin entanglement over long distances.
\end{abstract}

DOI: 10.1103/PhysRevLett.121.190401

Introduction.-In 1989, Franson conceived an interferometric setup to highlight the counter-intuitive implications of quantum mechanics [1]. He proposed to send a pair of entangled photons to two measurement stations (Alice and Bob), each composed of an unbalanced interferometer. By exploiting the quantum interference in the detection events, it should be possible to rule out local realistic models [2] by violating a Bell-Clauser-Horne-Shimony-Holt (CHSH) inequality [3]. Franson's idea was first implemented by exploiting energy-time entanglement, generated by pumping a nonlinear crystal with a continuous-wave (cw) laser [4-6]. The emitted photons are generated at the same instant, which is uncertain within the coherence time of the source, leading to indistinguishability in the alternative paths the photons will take in the measurement stations. Extending Franson's idea, time-bin (TB) entanglement was introduced in 1999 by Brendel et al. [7]: the cw laser is replaced by a pulsed laser shining the nonlinear crystal after passing through an unbalanced "pump" interferometer. Now, the photons can be emitted at two possible times, depending on the path taken by the pump pulse in the first interferometer [Fig. 1(a)]. Both energy-time and time-bin entanglement have been widely used to distribute entanglement over long distances [8-12] and to realize fiber-based cryptographic systems $[13,14]$, aiming for device-independent security [15-17], which requires the loophole-free violation of a Bell inequality [18-21].

However, Aerts et al. noted that Franson's Bell test is intrinsically affected by the postselection loophole (PSL)
[22], which is present independently of the other loopholes (e.g., locality and detection) that could affect local-realistic tests [23]. In fact, Alice and Bob should postselect only the indistinguishable events occurring within a coincidence window $\Delta \tau_{c}$, discarding those photons arriving at different times. When performing such postselection, there exists a local-hidden-variable (LHV) model reproducing the quantum predictions $[22,24]$. Indeed, a LHV model admits the local delays to depend on the local parameter $\left(\varphi_{A}\right.$ or $\left.\varphi_{B}\right)$, but Alice and Bob need to compare these delays to perform

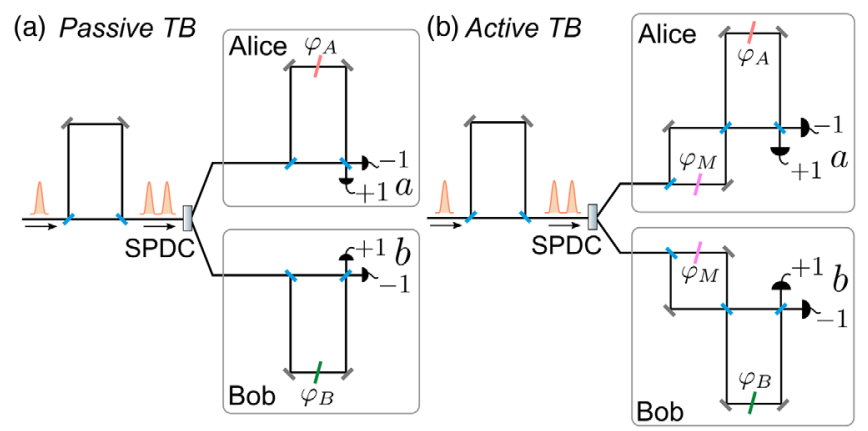

FIG. 1. Bell tests with time-bin entanglement. (a) In the passive $\mathrm{TB}$, by postselecting the events detected in coincidence in the central time slot, Alice and Bob can violate a Bell inequality, but the scheme is affected by the PSL. (b) In the active TB, the passive beam splitter is replaced by a balanced MZI acting as an optical switch, and a PSL-free Bell violation is obtained by exploiting the phase modulator $\varphi_{M}$ to not discard any data. 
the postselection. Therefore, even though the physical system is completely local, such postselection invalidates the locality assumption required to derive the Bell's inequality. The same loophole affects the time-bin entanglement scheme shown in Fig. 1(a), invalidating Bell's inequality as a test of local realism and enabling the hacking of Franson's scheme when used for cryptographic purposes [25]. In fact, the Bell test gives false evidence, since the apparent violation would tell users the setup is device-independently secure, while it is actually insecure because of the PSL.

Many modifications to Franson's original scheme have been proposed to address the PSL. The first one is due to Strekalov et al. [26], and exploited hyperentanglement in polarization and energy time to overcome the PSL by replacing the beam splitters of Alice's and Bob's interferometers with polarizing ones. This scheme was experimentally implemented [27] and recently realized in an intracity free-space link [28]. However, requiring entanglement in both energy time and polarization, this solution increases the experimental complexity. Afterwards, a proposal by Cabello et al. modified the geometry of the interferometers by interlocking them in a hug configuration, and introduced a local postselection, which does not require communication between Alice and Bob [29]. In this way, genuine energytime entanglement can be generated, i.e., not affected by the PSL. Soon after, table-top experiments were realized [30,31] as well as the distribution of genuine energy-time entanglement through $1 \mathrm{~km}$ of optical fibers [32] and its implementation in an optical fiber network [33]. However, the hug configuration requires to stabilize two long interferometers whose extension is determined by the distance between Alice and Bob: the larger the separation is, the more demanding the stabilization becomes. In the case of time-bin entanglement, the original proposal mentioned the use of active switches to prevent discarding any data [7], such as movable mirrors synchronized with the source, instead of passive beam splitters [Fig. 1(a)]. This solution can also be exploited to overcome the PSL [24], but no such scheme has been realized so far.

Here, we propose and implement, for the first time, a genuine time-bin entanglement scheme allowing the violation of a Bell's inequality free of the PSL. The active switches are realized by replacing the first beam splitter, in each unbalanced interferometer of the measurement stations, with another balanced interferometer with a fast phase shifter in one arm [Fig. 1(b)]. By actively synchronizing the phase shifter with the pump pulses, it is possible to use the full detection statistics, overcoming the PSL. The independence between Alice's and Bob's terminals, the relaxed stabilization requirements, as well as the compliance with off-the-shelf components open the possibility to exploit such scheme over long distances, paving the way to a conclusive loophole-free Bell test with time-bin entanglement. Indeed, the postselection procedure has long precluded the use of time-bin entanglement in loophole-free Bell experiments, performed, so far, by exploiting electronic spins [18], atoms [19], and photon polarization [20,21].

Conceptual analysis of time-bin entanglement schemes.In the passive time-bin scheme, a pump Mach-Zehnder interferometer (MZI) with a temporal imbalance equal to $\Delta t$ is used to split a short light pulse into two [Fig. 1(a)]. This light is focused into a nonlinear crystal producing photon pairs via a spontaneous parametric down-conversion (SPDC) process. By optimizing the pump energy, the generation of double photon pairs is suppressed, and the Bell state $\left|\Phi^{+}\right\rangle=\left(|S\rangle_{A}|S\rangle_{B}+|L\rangle_{A}|L\rangle_{B}\right) / \sqrt{2}$ is produced, where the indexes $A$ and $B$ represent the generated photons that are sent to Alice's and Bob's measurement stations. Each of these is composed of an unbalanced MZI that has the same imbalance $\Delta t$ of the pump interferometer and can introduce a further phase shift $\varphi_{A}, \varphi_{B}$. The output ports of each interferometer are followed by two single-photon detectors, and the possible outcomes are labeled $a= \pm 1$ and $b= \pm 1$ for Alice and Bob, respectively.

In the passive TB scheme, each photon of the pair can be detected only at three distinct times $\left(t_{0}-\Delta t, t_{0}, t_{0}+\Delta t\right)$, due to the pump and measurement MZIs. By postselecting the detection events occurring in the central time slot, Alice realizes the projection $\left\{\hat{P}_{a}\right\}_{a= \pm 1}$ with $\hat{P}_{a}=\left|\psi_{a}\right\rangle\left\langle\psi_{a}\right|$ where $\left|\psi_{a}\right\rangle=\left(|S\rangle+a e^{i \varphi_{A}}|L\rangle\right) / \sqrt{2}$, and similar relations hold for Bob (with $a$ replaced by $b$ and $A$ by $B$ ). Since the delay is local, one could think that this should allow the violation of the Bell's inequality. There is simply no physical mechanism for the remote phase shift to influence the local delay. However, for a coincidence to occur, Bob's delay needs to coincide with Alice's, and Bob's delay is controlled by Bob's phase shift, remotely from the point of view of Alice. This constitutes a coincidence loophole for the Bell inequality [34], somewhat similar to a detection loophole with 50\% detection efficiency, but much worse since it is present even when using loss-free equipment, therefore, introducing an unavoidable intrinsic loophole in the setup.

Quantum mechanics provides the probabilities $\mathcal{P}_{a, b}$ for photon detections that occur within a coincidence window $\Delta \tau_{c}<\Delta t$ around the central time slot for each pair of detectors $a, b$. The probabilities $\mathcal{P}_{a, b}$ depend on the initial state $\left|\Phi^{+}\right\rangle$and on the local phase shifts $\varphi_{A}, \varphi_{B}$ introduced by the measurement stations and are given by $\mathcal{P}_{a, b}\left(\varphi_{A}, \varphi_{B}\right)=\frac{1}{4}\left[1+a b \mathcal{V} \cos \left(\varphi_{A}+\varphi_{B}\right)\right]$, where $\mathcal{V}$ is the visibility of two-photon interference.

Disregarding the PSL, the interference in the postselected events will seem to violate the Bell-CHSH inequality, which provides an upper limit for a combination of four correlation functions $E\left(\varphi_{A}, \varphi_{B}\right)$ with different phases $\varphi_{A}, \varphi_{B}$, when assuming the existence of a LHV model [3]. The correlation function is $E\left(\varphi_{A}, \varphi_{B}\right)=\sum_{a, b} a b \mathcal{P}_{a, b}\left(\varphi_{A}, \varphi_{B}\right)$ and the BellCHSH inequality $S \leq 2$ is given by the $S$ parameter $S \equiv E\left(\varphi_{A}, \varphi_{B}\right)+E\left(\varphi_{A}^{\prime}, \varphi_{B}\right)+E\left(\varphi_{A}, \varphi_{B}^{\prime}\right)-E\left(\varphi_{A}^{\prime}, \varphi_{B}^{\prime}\right)$, where $\varphi_{A}, \varphi_{A}^{\prime}$ and $\varphi_{B}, \varphi_{B}^{\prime}$ denote the values of the phase shifts 

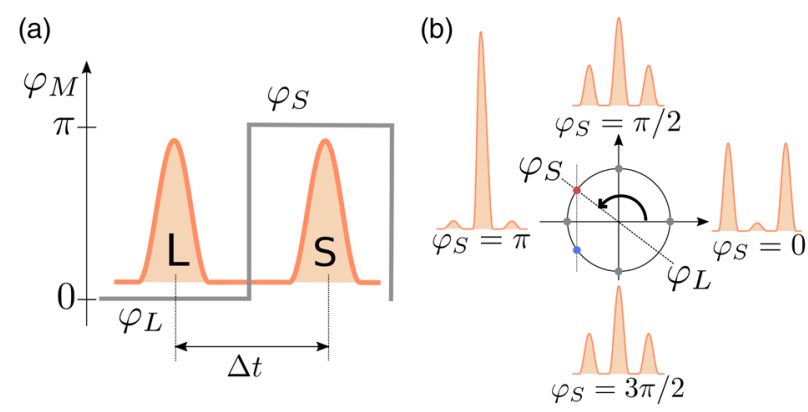

FIG. 2. Functioning of the active TB scheme. (a) In a balanced MZI, the relative phase $\varphi_{M}$ sensed by a traveling pulse determines the output port at which it will exit with probabilities $\cos ^{2}\left(\varphi_{M} / 2\right)$ and $\sin ^{2}\left(\varphi_{M} / 2\right)$. A fast modulator imposes different phase shifts $\varphi_{S}$ and $\varphi_{L}$ to the $|S\rangle$ and $|L\rangle$ photons while they are traveling along the balanced MZI. (b) The detection pattern depends on $\varphi_{S}$ and $\varphi_{L}=\varphi_{S}-\pi$. If $\varphi_{S}=\pi$, all detections occur in the central time slot; if $\varphi_{S}=0$, they appear only in the lateral time slots. Any other detection histogram can be obtained with two different $\varphi_{S}$ values, one with $\varphi_{S}<\pi$ (red dot) and the other with $\varphi_{S}>\pi$ (blue dot).

introduced by Alice and Bob, respectively [3]. Quantum mechanics $(\mathrm{QM})$ predicts $E^{\mathrm{QM}}\left(\varphi_{A}, \varphi_{B}\right)=\mathcal{V} \cos \left(\varphi_{A}+\varphi_{B}\right)$, leading to a maximum $S$ parameter $S_{\max }=2 \sqrt{2} \mathcal{V}$ if $\varphi_{A}=$ $-\pi / 4, \varphi_{A}^{\prime}=\pi / 4, \varphi_{B}=0$, and $\varphi_{B}^{\prime}=\pi / 2$. Hence, the Bell-CHSH inequality will seem to be violated if $\mathcal{V}>1 / \sqrt{2} \approx 0.71$.

Moreover, if no postselection is applied in the passive TB scheme, then the Bell-CHSH inequality holds, and could, in principle, be violated. However, in this case, Alice implements the positive operator valued measure (POVM) [35] $\left\{\hat{\Gamma}_{a}\right\}_{a= \pm 1}$ with $\hat{\Gamma}_{a}=(1 / 4) \mathbb{1}+(1 / 2) \hat{P}_{a}$, where $\mathbb{1}=$ $|S\rangle\langle S|+| L\rangle\langle L|$ (and, similarly, for Bob). Thus, with no postselection, the quantum detection probabilities $\mathcal{P}_{a, b}$ lead to $S_{\max }=2 \sqrt{2} \mathcal{V}^{\prime}$, with the overall three-peak visibility $\mathcal{V}^{\prime}=\mathcal{V} / 4$ and the Bell-CHSH inequality cannot be violated even with perfect visibility $\mathcal{V}=1$.

Contrarily, a proper violation can be achieved in the active TB scheme here proposed [Fig. 1(b)]. We replace the passive beam splitter with an additional balanced MZI acting as a fast optical switch, allowing the measurement MZI to recombine the $|S\rangle$ and $|L\rangle$ pulses, making them indistinguishable. Contrary to the passive TB scheme which recombines the two temporal modes in a probabilistic manner, our scheme deterministically compensates for the delay $\Delta t$, and no detections are discarded. Indeed, by imposing the phases $\varphi_{S}$ and $\varphi_{L}=\varphi_{S}-\pi$ on the $|S\rangle$ and $|L\rangle$ pulses, respectively, the balanced MZI determines the path they will take in the measurement-MZI, as sketched in Fig. 2(a).

At each detector, we expect a detection pattern that depends on the value of $\varphi_{S}$ [Fig. 2(b)]. In our active TB scheme, Alice implements the POVM $\left\{\hat{\Pi}_{a}\right\}_{a= \pm 1}$, where $\hat{\Pi}_{a}=$ $\frac{1}{2}\left[\cos ^{2}\left(\varphi_{S} / 2\right)|S\rangle\left\langle S\left|+\sin ^{2}\left(\varphi_{L} / 2\right)\right| L\right\rangle\langle L|\right]+\left|\chi_{a}\right\rangle\left\langle\chi_{a}\right| \quad$ with

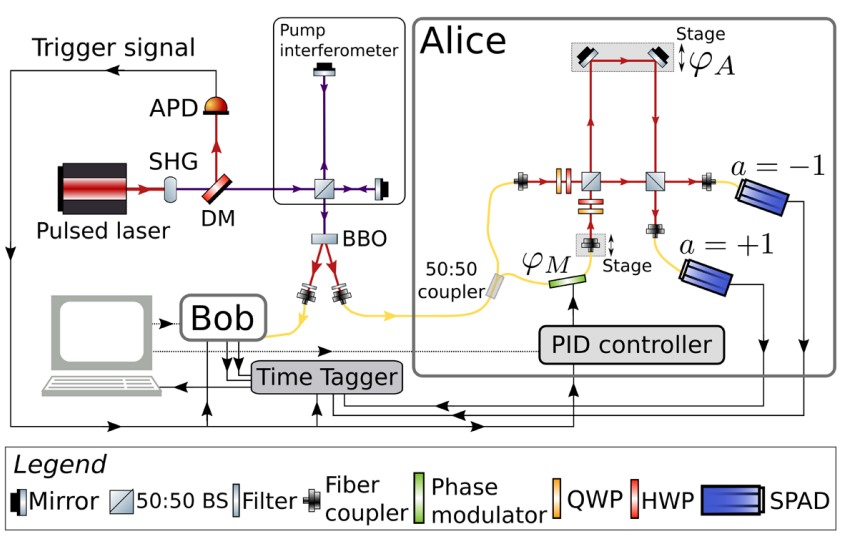

FIG. 3. Experimental setup. Bob's measurement station is analogue to Alice's. APD: analog photodetector; DM: dichroic mirror; HWP: half waveplate; and QWP: quarter waveplate.

$\left|\chi_{a}\right\rangle=\left[i e^{-i\left(\varphi_{S} / 2\right)} \sin \left(\varphi_{S} / 2\right)|S\rangle+a e^{i\left(\varphi_{A}-\varphi_{L} / 2\right)} \cos \left(\varphi_{L} / 2\right)|L\rangle\right] /$ $\sqrt{2}$. If $\varphi_{S}-\varphi_{L}=\pi$, we have

$$
\hat{\Pi}_{a}=\frac{1}{2} \cos ^{2}\left(\frac{\varphi_{S}}{2}\right) \mathbb{1}+\sin ^{2}\left(\frac{\varphi_{S}}{2}\right) \hat{P}_{a} .
$$

Setting the phase $\varphi_{S}=\pi$ (so $\varphi_{L}=0$ ), $\hat{\Pi}_{a}$ reduces to $\hat{P}_{a}$ and she projects onto the state $\left|\psi_{a}\right\rangle$, with no postselection procedure. Indeed, in the detection pattern, the lateral peaks "disappear" [Fig. 2(b)], and it is unnecessary to discard any data. Hence, the expected violation of Bell-CHSH inequality is PSL free.

Description of the experiment.-We implemented the active TB scheme proposed above by using the experimental setup sketched in Fig. 3. A mode-locking laser produced a pulse train with wavelength around $808 \mathrm{~nm}$, $76 \mathrm{MHz}$ of repetition rate and $\sim 150 \mathrm{fs}$ of pulse duration. This beam is used to pump a second-harmonic-generation (SHG) crystal generating coherent pulses of light upconverted to $404 \mathrm{~nm}$. Each pulse passes through a freespace unbalanced Michelson interferometer (the pump interferometer), producing a coherent state in two temporal modes. The imbalance between the two arms is about $90 \mathrm{~cm}$, corresponding to a temporal imbalance $\Delta t \approx 3 \mathrm{~ns}$, much greater than the coherence time of the pulses. Then, the pulses pump a 2-mm long beta-barium borate (BBO) crystal to produce the entangled photon state at $808 \mathrm{~nm}$ via type-II SPDC [36].

The two photons are sent to Alice's and Bob's terminals after being spectrally filtered (3 $\mathrm{nm}$ bandwidth) and collected by two single-mode optical fibers. Each station is composed of two MZIs, a balanced one and an unbalanced one. The balanced MZI is composed of a 50:50 fiber coupler which defines the two arms of the interferometer. To guarantee the zero imbalance of this MZI, a nanometric stage is placed in one of the two arms. The balanced MZI works as a fast optical switch, since there is a fast ( $\sim \mathrm{GHz}$ bandwidth) phase modulator in one of 


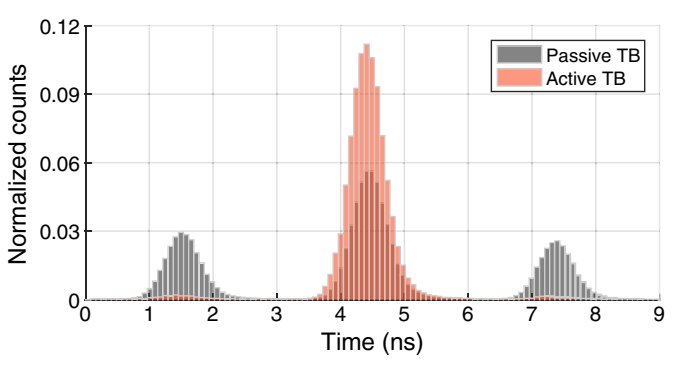

FIG. 4. Raw detection histograms obtained with one detector for the passive TB (grey curve) and with the active TB (red curve). The counts are normalized to fairly compare the two histograms.

its arms. The modulation voltage is set to $V_{\pi}$ such that $\varphi_{S}-\varphi_{L}=\pi$, while the dc bias of the phase modulator is driven by an external proportional-integral-derivative (PID) controller, that is responsible for locking the phase $\varphi_{S}$ to $\pi$. The complete operating principle of the PID controller is detailed in the Supplemental Material (SM) [37]. The two arms of the balanced MZI are recombined at a 50:50 freespace beam splitter (BS) after it has been optimized for polarization rotations. This BS begins the unbalanced MZI whose imbalance is equal to that of the pump interferometer (within the coherence time $\sim 200 \mu$ s of the photons). The two mirrors of the long arm of the unbalanced MZI are placed on a nanometric piezoelectric stage to both guarantee the required imbalance $\Delta t$ and introduce the local phase shift $\varphi_{A}$ and $\varphi_{B}$ to realize the Bell test. At the two output ports of the measurement stations, we used two avalanche single photon detectors (SPADs, $~ 50 \%$ detection efficiency). The detection events are then time tagged by a time-to-digital converter (time tagger) with 81 ps resolution and the data are stored in a personal computer.

Results.-With the setup shown in Fig. 3, we performed three different Bell tests: (I) the passive TB with postselection, (II) the passive TB with no postselection, (III) the active TB with no postselection proposed above. To realize (I), we bypassed the balanced MZI in each of the measurement stations, hence, obtaining the passive TB configuration of Fig. 1(a). By choosing a coincidence window $\Delta \tau_{c} \approx 2.4 \mathrm{~ns}$ and by postselecting the coincident events that occurred in the central time slot, Alice (Bob) implemented $\hat{P}_{a}\left(\hat{P}_{b}\right)$, and the expected Bell-CHSH violation is affected by the PSL. To realize (II), we used the same configuration as in (I), without discarding any data by choosing a coincidence window $\Delta \tau_{c} \approx 8.1 \mathrm{~ns}$, as large as the three-peak profile in the detections (Fig. 4). Now, Alice (Bob) implemented the POVM $\hat{\Gamma}_{a}\left(\hat{\Gamma}_{b}\right)$ and no Bell-CHSH violation is expected. To implement (III), we exploited the balanced MZI in each station, and we used the PID controller to implement the phase-locking mechanism independently at each terminal. We did not discard any data by choosing a large coincidence window as in (II), and the Bell-CHSH inequality is directly applicable, since Alice
TABLE I. Main results. SD refers to standard deviation of the Bell-CHSH violation.

\begin{tabular}{lccccc}
\hline \hline Scheme & $\Delta \tau_{c}(\mathrm{~ns})$ & PSL & $\mathcal{V}_{\exp }$ & $S_{\exp }$ & SD \\
\hline (I) passive TB & 2.4 & Yes & $0.95 \pm 0.05$ & $2.58 \pm 0.03$ & 18.3 \\
(II) passive TB & 8.1 & No & $0.23 \pm 0.02$ & $0.67 \pm 0.02$ & $\ldots$ \\
(III) active TB & 8.1 & No & $0.89 \pm 0.03$ & $2.30 \pm 0.03$ & 9.3 \\
\hline \hline
\end{tabular}

(and Bob) implemented the POVM of Eq. (1) with $\varphi_{S}=\pi$. Hence, the expected Bell-CHSH violation is free of the PSL.

A typical histogram from a detector is shown in Fig. 4, being similar to the results from the other ones. For the TB schemes (I) and (II), as the balanced MZI is bypassed, the expected three-peak profile (grey histogram) is found. Conversely, in our active TB scheme (III), the PID controller correctly clears the lateral peaks (red histogram).

For each of the Bell tests described above, first, we calibrated the shifts to be introduced by the nanometric stages in Alice's and Bob's unbalanced MZIs, by scanning the coincidence rate for a pair of detectors by moving Bob's stage while Alice's is fixed. From the sinusoidal pattern so obtained, we estimated the experimental visibility $\mathcal{V}_{\exp }$ for each scheme. Then, we imposed the shifts $\left(\varphi_{A}, \varphi_{B}\right)$ needed to obtain the maximal violation of the Bell inequality and run the acquisition long enough for significant statistics.

The results of the tests are presented in Table I. Violations of the Bell-CHSH inequality were obtained with the first and the third scheme with clear statistical evidence, but only (III) is not affected by the PSL. The minor violation obtained is due to imperfection in the balanced MZI alignment and in the locking procedure during the data acquisitions needed to experimentally estimate the $S$ parameter $S_{\text {exp }}$. However, any imperfection in the locking mechanism setting $\varphi_{S}=\pi$ corresponds to an effective lower visibility, but it does not introduce any loophole in the Bell test.

Conclusions.-Time-bin encoding [7] is a resource for performing fundamental tests of quantum mechanics [38,39], quantum communications over turbulent channels $[40,41]$, and for distributing entanglement over long distances [12]. Time bin is more robust than polarization in fiber-based implementations, since the latter suffers from polarization mode dispersion [14], that must be carefully compensated [42]. Recently, a three-state quantum key distribution (QKD) protocol has been proposed and implemented using both encodings [43,44]. The reduced complexity of time bin allowed for a higher secret key rate and the longest fiber QKD link realized so far [45]. Furthermore, a significant advantage of time bin is the possibility of realizing high-dimensional states (qudits), which can provide higher QKD rates [46,47], and that can be controlled (see the proposal [48]) by exploiting the square phase modulation we experimentally realized here.

However, all time-bin entanglement realizations performed so far were affected by the PSL. Another possible 
way to overcome it requires violating the "chained" Bell inequalities [49], but the needed visibility ( $\gtrsim 0.94$ [25]) is considerably higher than that of the Bell-CHSH inequality $(\gtrsim 0.71)$. Even if such a high visibility is achievable [50], our scheme strongly relaxes this requirement, since the Bell-CHSH inequality is directly applicable.

This work is the first implementation of genuine time-bin entanglement, representing a crucial step towards its exploitation for fundamental tests of physics and the realization of the quantum internet [51]. Our scheme can be realized using only commercial off-the-shelf fiber components and, since its stability does not depend on the distance between Alice and Bob, it is easier to be implemented with respect to the hug configuration [29]. Furthermore, as long as both the $\pi$-phase transition imposed by the modulator and the detector jitter are shorter than the imbalance, it is possible to shorten $\Delta \mathrm{t}$, rendering it compatible with today's photonic integrated technologies $[52,53]$. Finally, our work makes time-bin entanglement a viable technique for obtaining a loophole-free Bell violation, which is the enabling ingredient of any deviceindependent protocol [15-17,54].

[1] J. D. Franson, Bell Inequality for Position and Time, Phys. Rev. Lett. 62, 2205 (1989).

[2] J. S. Bell, On the Einstein Podolsky Rosen paradox, Physics 1, 195 (1964).

[3] J. F. Clauser, M. A. Horne, A. Shimony, and R. A. Holt, Proposed Experiment to Test Local Hidden-Variable Theories, Phys. Rev. Lett. 23, 880 (1969).

[4] Z. Y. Ou, X. Y. Zou, L. J. Wang, and L. Mandel, Observation of Nonlocal Interference in Separated Photon Channels, Phys. Rev. Lett. 65, 321 (1990).

[5] J. Brendel, E. Mohler, and W. Martienssen, Experimental test of Bell's inequality for energy and time, Europhys. Lett. 20, 575 (1992).

[6] P. G. Kwiat, A. M. Steinberg, and R. Y. Chiao, Highvisibility interference in a Bell-inequality experiment for energy and time, Phys. Rev. A 47, R2472(R) (1993).

[7] J. Brendel, N. Gisin, W. Tittel, and H. Zbinden, Pulsed Energy-Time Entangled Twin-Photon Source for Quantum Communication, Phys. Rev. Lett. 82, 2594 (1999).

[8] P. R. Tapster, J. G. Rarity, and P. C. M. Owens, Violation of Bell's Inequality over 4 km of Optical Fiber, Phys. Rev. Lett. 73, 1923 (1994).

[9] W. Tittel, J. Brendel, H. Zbinden, and N. Gisin, Violation of Bell Inequalities by Photons More than $10 \mathrm{~km}$ Apart, Phys. Rev. Lett. 81, 3563 (1998).

[10] W. Tittel, J. Brendel, N. Gisin, and H. Zbinden, Longdistance Bell-type tests using energy-time entangled photons, Phys. Rev. A 59, 4150 (1999).

[11] I. Marcikic, H. de Riedmatten, W. Tittel, H. Zbinden, M. Legré, and N. Gisin, Distribution of Time-Bin Entangled Qubits over $50 \mathrm{~km}$ of Optical Fiber, Phys. Rev. Lett. 93, 180502 (2004).
[12] T. Inagaki, N. Matsuda, O. Tadanaga, M. Asobe, and H. Takesue, Entanglement distribution over $300 \mathrm{~km}$ of fiber, Opt. Express 21, 23241 (2013).

[13] W. Tittel, J. Brendel, H. Zbinden, and N. Gisin, Quantum Cryptography Using Entangled Photons in Energy-Time Bell States, Phys. Rev. Lett. 84, 4737 (2000).

[14] N. Gisin, G. Ribordy, W. Tittel, and H. Zbinden, Quantum cryptography, Rev. Mod. Phys. 74, 145 (2002).

[15] A. Acín, N. Gisin, and L. Masanes, From Bell's Theorem to Secure Quantum Key Distribution, Phys. Rev. Lett. 97, 120405 (2006).

[16] A. Acín, N. Brunner, N. Gisin, S. Massar, S. Pironio, and V. Scarani, Device-Independent Security of Quantum Cryptography Against Collective Attacks, Phys. Rev. Lett. 98, 230501 (2007).

[17] R. Arnon-Friedman, F. Dupuis, O. Fawzi, R. Renner, and T. Vidick, Practical device-independent quantum cryptography via entropy accumulation, Nat. Commun. 9, 459 (2018).

[18] B. Hensen, H. Bernien, A. E. Dréau, A. Reiserer, N. Kalb, M. S. Blok, J. Ruitenberg, R. F. L. Vermeulen, R. N. Schouten, C. Abellán, W. Amaya, V. Pruneri, M. W. Mitchell, M. Markham, D. J. Twitchen, D. Elkouss, S. Wehner, T. H. Taminiau, and R. Hanson, Loophole-free Bell inequality violation using electron spins separated by 1.3 kilometres, Nature (London) 526, 682 (2015).

[19] W. Rosenfeld, D. Burchardt, R. Garthoff, K. Redeker, N. Ortegel, M. Rau, and H. Weinfurter, Event-Ready Bell Test Using Entangled Atoms Simultaneously Closing Detection and Locality Loopholes, Phys. Rev. Lett. 119, 010402 (2017).

[20] M. Giustina et al., Significant-Loophole-Free Test of Bell's Theorem with Entangled Photons, Phys. Rev. Lett. 115, 250401 (2015).

[21] L. K. Shalm et al., Strong Loophole-Free Test of Local Realism, Phys. Rev. Lett. 115, 250402 (2015).

[22] S. Aerts, P. Kwiat, J.-Å. Larsson, and M. Żukowski, TwoPhoton Franson-Type Experiments and Local Realism, Phys. Rev. Lett. 83, 2872 (1999).

[23] J.-Å. Larsson, Loopholes in Bell inequality tests of local realism, J. Phys. A 47, 424003 (2014).

[24] J. Jogenfors and J.-A. Larsson, Energy-time entanglement, elements of reality, and local realism, J. Phys. A 47, 424032 (2014).

[25] J. Jogenfors, A. M. Elhassan, J. Ahrens, M. Bourennane, and J.-A. Larsson, Hacking the Bell test using classical light in energy-time entanglement-based quantum key distribution, Sci. Adv. 1, e1500793 (2015).

[26] D. V. Strekalov, T. B. Pittman, A. V. Sergienko, Y. H. Shih, and P. G. Kwiat, Postselection-free energy-time entanglement, Phys. Rev. A 54, R1(R) (1996).

[27] J. T. Barreiro, N. K. Langford, N. A. Peters, and P. G. Kwiat, Generation of Hyperentangled Photon Pairs, Phys. Rev. Lett. 95, 260501 (2005).

[28] F. Steinlechner, S. Ecker, M. Fink, B. Liu, J. Bavaresco, M. Huber, T. Scheidl, and R. Ursin, Distribution of highdimensional entanglement via an intra-city free-space link, Nat. Commun. 8, 15971 (2017).

[29] A. Cabello, A. Rossi, G. Vallone, F. De Martini, and P. Mataloni, Proposed Bell Experiment with Genuine EnergyTime Entanglement, Phys. Rev. Lett. 102, 040401 (2009). 
[30] G. Lima, G. Vallone, A. Chiuri, A. Cabello, and P. Mataloni, Experimental Bell-inequality violation without the postselection loophole, Phys. Rev. A 81, 040101 (2010).

[31] G. Vallone, I. Gianani, E. B. Inostroza, C. Saavedra, G. Lima, A. Cabello, and P. Mataloni, Testing Hardy's nonlocality proof with genuine energy-time entanglement, Phys. Rev. A 83, 042105 (2011).

[32] Á. Cuevas, G. Carvacho, G. Saavedra, J. Cariñe, W. A. T. Nogueira, M. Figueroa, A. Cabello, P. Mataloni, G. Lima, and G. B. Xavier, Long-distance distribution of genuine energy-time entanglement, Nat. Commun. 4, 2871 (2013).

[33] G. Carvacho, J. Cariñe, G. Saavedra, Á. Cuevas, J. Fuenzalida, F. Toledo, M. Figueroa, A. Cabello, J.-Å. Larsson, P. Mataloni, G. Lima, and G. B. Xavier, Postselection-Loophole-Free Bell Test over an Installed Optical Fiber Network, Phys. Rev. Lett. 115, 030503 (2015).

[34] J.-A. Larsson and R. D. Gill, Bell's inequality and the coincidence-time loophole, Europhys. Lett. 67, 707 (2004).

[35] A. Peres, Quantum Theory: Concepts and Methods (Kluwer Academic Publishers, Dordrecht, 1993).

[36] P. G. Kwiat, K. Mattle, H. Weinfurter, A. Zeilinger, A. V. Sergienko, and Y. Shih, New High-Intensity Source of Polarization-Entangled Photon Pairs, Phys. Rev. Lett. 75, 4337 (1995).

[37] See Supplemental Material at http://link.aps.org/ supplemental/10.1103/PhysRevLett.121.190401 for the detailed description of the PID controller.

[38] I. Marcikic, H. de Riedmatten, W. Tittel, H. Zbinden, and N. Gisin, Long-distance teleportation of qubits at telecommunication wavelengths, Nature (London) 421, 509 (2003).

[39] F. Vedovato, C. Agnesi, M. Schiavon, D. Dequal, L. Calderaro, M. Tomasin, D. G. Marangon, A. Stanco, V. Luceri, G. Bianco, G. Vallone, and P. Villoresi, Extending Wheelers delayed-choice experiment to space, Sci. Adv. 3, e1701180 (2017).

[40] G. Vallone, D. Dequal, M. Tomasin, F. Vedovato, M. Schiavon, V. Luceri, G. Bianco, and P. Villoresi, Interference at the Single Photon Level Along Satellite-Ground Channels, Phys. Rev. Lett. 116, 253601 (2016).

[41] J. Jin, S. Agne, J.-P. Bourgoin, Y. Zhang, N. Lütkenhaus, and T. Jennewein, Demonstration of analyzers for multimode photonic time-bin qubits, Phys. Rev. A 97, 043847 (2018).

[42] H. Hübel, M. R. Vanner, T. Lederer, B. Blauensteiner, T. Lorünser, A. Poppe, and A. Zeilinger, High-fidelity transmission of polarization encoded qubits from an entangled source over $100 \mathrm{~km}$ of fiber, Opt. Express 15, 7853 (2007).

[43] F. Grünenfelder, A. Boaron, D. Rusca, A. Martin, and H. Zbinden, Simple and high-speed polarization-based QKD, Appl. Phys. Lett. 112, 051108 (2018).

[44] A. Boaron, B. Korzh, R. Houlmann, G. Boso, D. Rusca, S. Gray, M.-J. Li, D. Nolan, A. Martin, and H. Zbinden, Simple 2.5 GHz time-bin quantum key distribution, Appl. Phys. Lett. 112, 171108 (2018).

[45] A. Boaron, G. Boso, D. Rusca, C. Vulliez, C. Autebert, M. Caloz, M. Perrenoud, G. Gras, F. Bussières, M.-J. Li, D. Nolan, A. Martin, and H. Zbinden, Secure Quantum Key Distribution Over $421 \mathrm{~km}$ of Optical Fiber, arXiv: 1807.03222 [Phys. Rev. Lett. (to be published)].

[46] T. Brougham, S. M. Barnett, K. T. McCusker, P. G. Kwiat, and D. J. Gauthier, Security of high-dimensional quantum key distribution protocols using Franson interferometers, J. Phys. B 46, 104010 (2013).

[47] N. T. Islam, C. C. W. Lim, C. Cahall, J. Kim, and D. J. Gauthier, Provably secure and high-rate quantum key distribution with time-bin qudits, Sci. Adv. 3, e1701491 (2017).

[48] J. M. Lukens, N. T. Islam, C. C. W. Lim, and D. J. Gauthier, Reconfigurable generation and measurement of mutually unbiased bases for time-bin qudits, Appl. Phys. Lett. 112, 111102 (2018).

[49] S. Braunstein and C. M. Caves, Wringing out better Bell inequalities, Ann. Phys. (N.Y.) 202, 22 (1990).

[50] M. Tomasin, E. Mantoan, J. Jogenfors, G. Vallone, J.-A. Larsson, and P. Villoresi, High-visibility time-bin entanglement for testing chained Bell inequalities, Phys. Rev. A 95, 032107 (2017).

[51] H. J. Kimble, The quantum internet, Nature (London) 453, 1023 (2008).

[52] P. Sibson, J. E. Kennard, S. Stanisic, C Erven, J. L. O’Brien, and M.G. Thompson, Integrated silicon photonics for high-speed quantum key distribution, Optica 4, 172 (2017).

[53] V. Sorianello, M. Midrio, G. Contestabile, I. Asselberghs, J. Van Campenhout, C. Huyghebaert, I. Goykhman, A. K. Ott, A. C. Ferrari, and M. Romagnoli, Graphene-silicon phase modulators with gigahertz bandwidth, Nat. Photonics 12, 40 (2018).

[54] A. Acín and L. Masanes, Certified randomness in quantum physics, Nature (London) 540, 213 (2016). 